\title{
Improving the Content of a Client-server Technology Training Course: Set up and Collaborative Implementation of Local and Cloud-based Remote Servers
}

\author{
https://doi.org/10.3991/ijet.v14i21.10643 \\ Meruert Serik( $\left.{ }^{\bowtie}\right)$, Meiramgul Mukhambetova \\ L.N. Gumilyev Eurasian National University, Nur-Sultan, Kazakhstan \\ serik_meruerts@mail.ru \\ Alibek Yeskermessuly \\ Arkalyk State Pedagogical Institute named after Y.Altynsarin, \\ Arkalyk, Kazakhstan
}

\begin{abstract}
The integration of software solutions in the content of training courses resulted from the socio-economic demand, has a positive effect on the enhancement of trainee students for future professional activities. However, the latest achievements of hardware and software are still not included in the content of the higher education in a timely manner. Therefore, we focused in our research on the improvement of the content of the training course based on a client-server technology by using modern software capabilities.

The article presents the conceptual structure of the training course, settings and the integration of local and public cloud-based servers. It describes approaches of its use in the learning process. Microsoft SQL Server 2016, Microsoft Azure SQL Database, ASP.NET MVC 5 Framework were used for teaching environments. The new course was introduced for Computer Science students of L.N.Gumilyov Eurasian National University and Kh.Dosmukhamedov Atyrau State University in Kazakhstan.
\end{abstract}

Keywords-client-server, training course, local server, cloud-based server, Microsoft Azure SQL Database, hybrid cloud principle.

\section{Introduction}

Rapidly evolving socio-economic processes increase needs for data processing and access. Increasing the amount of data processed and requirement for access has led to the emergence of systems such as distributed and enterprise computing. A clientserver technology is used in the creation of online systems as an effective source of data processing to improve the quality of knowledge in the academic fields, marketing and services of many businesses and companies. 
Paper-Improving the Content of a Client-server Technology Training Course: Set up and Collaborative...

The possibility of using client-server model with the modern capabilities of hardware and software for revision requires the following conditions. Despite the excessive number of mobile and other computing platforms, one of them is providing a convenient and free access for personal and professional data [1]. Currently, clouds computing, as a new model of representing the supply of computing resources takes the leading position. Nikulchev, E., Pluzhnik, E., Biryukov, D., Lukyanchikov, O.[2, p.963-964] in their work considered: "Cloud system is a typical client-server system that is focused on servicing a large number of customers".

Many companies and social organizations, in accordance with the new trends, upgrade the traditional client-server systems, showing interest in migration and integration with the cloud. Some enterprises have already deployed a server of their platforms based on the cloud. Meanwhile, contradictions between employers' demand and educational programs can lead to obstacles in the employment of graduates.

The purpose of the study: to provide training for future professional activities through improving the content of the training course based on the client-server technology. Thus, analyzing the state of training courses on the client-server technology in Kazakhstan and other countries [3], we have found that there were cases when the content of them was studied using the old program systems.The content of the training course developed by us is carried out in several stages through the following technological processes:

1) organising of the client-server system operation by setting MS SQL Server, and client settings in the local network of the University;

2) deploying a server in the cloud and creating a database on the configured server using the Microsoft Azure SQL Database resource, implementing access to the databases from the client computer;

3) setting up a local server MS SQL at University and Microsoft Azure SQL Database server, using the hybrid cloud principle in their communication;

4) creation of web applications linked with the servers in ASP.NET MVC 5 Framework.

The training course with the improved content was held for the educational programs in Computer Science in L.N.Gumilyov Eurasian National University and Kh.Dosmukhamedov Atyrau State University.

\section{Literature review}

The enhancement of the methodological system content of the training course is a part of the modern concept of improving the students' training[4]. The course on the client-server technologies is considered both as an academic understanding and reasonable level of practical knowledge of the concepts and principles of the clientserver architecture, taking into account the migration from the legacy systems [5].

The emergence of the cloud computing technology brings a new information computing infrastructure. Databases, storage, and computing utilities are available as services. The key feature is that it hides the main complexity of the use of IT resources while providing scalable, reliable and sustainable use of cost-effective 
services [6], [7], [8]. Let's focus on the systems designed for education to highlight the practical need of the technologies that served the basis of our research.

The following works described the system of modeling accounting skills [9], cloud-based English educational platform [10], cloud storage for the students of Computer Science [11]. In Reference [12], the function of hybrid cloud interface for data analysis tics in the education sector was described. Hybrid cloud, implemented on the basis of the platform modification effectively serves in the work with a large volume of databases [13].

\section{Training Course Structure Improvement}

This section describes the conceptual structure of the course content which reflects the cohesion of topics. In addition, a brief description of software environments was designed to implement the structure of the content.

\subsection{Training course structure}

The training course developed in the new structure provides an opportunity along with the concepts of creating traditional client-server systems and using public cloud resources to be familiar with the principles of hybrid cloud configuration through the platform modification. The figure presented below shows the structure of the improved content training course (Figure 1).

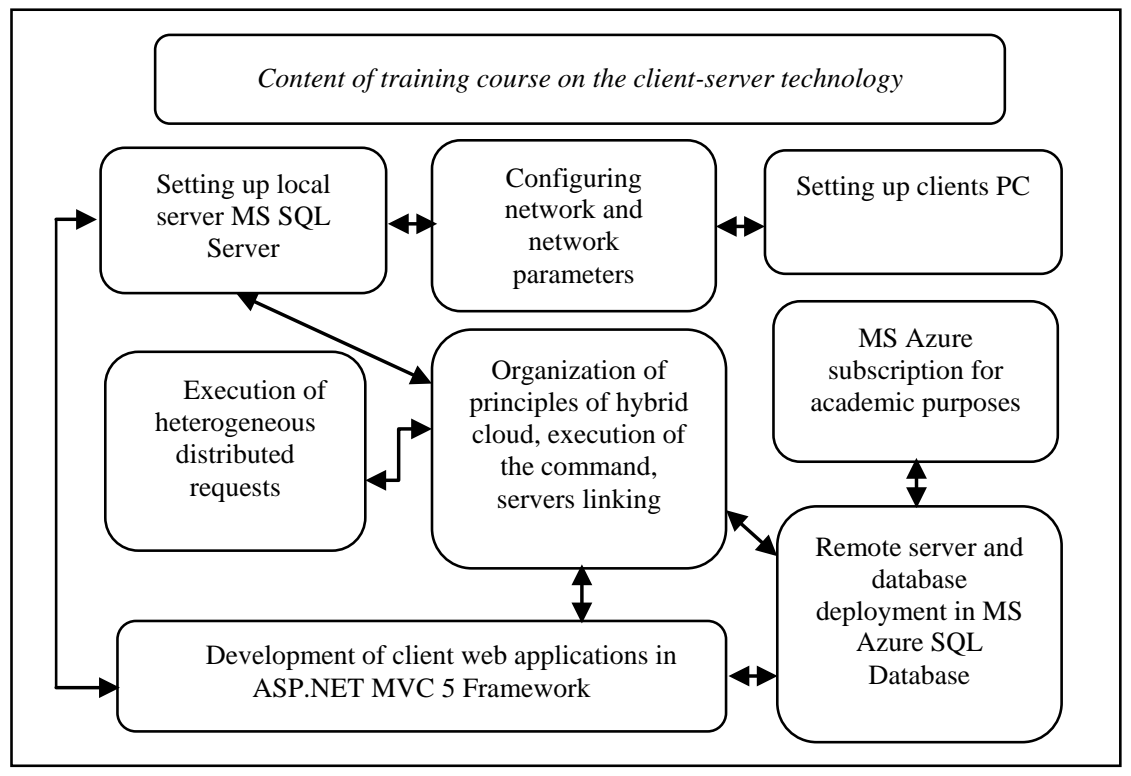

Fig. 1. Structure of training course 


\subsection{Software development environments and cloud platforms used in the training course process}

MS SQL Server 2016: MS SQL Server 2016 is the latest addition to the Microsoft data platform with many new features and improvements that provide the breakthrough performance, enhanced security, more feature-rich, integrated reporting and analytics capabilities. Built SQL Server 2016, using the new quick release model, includes many features that have been first introduced in the cloud of the Microsoft Azure SQL database. In addition, SQL Server 2016 includes the ability of dynamic transfer of the historical data to the cloud [14].The version of MS SQL Server Express has free environment. Therefore, the program is available for students and all the users.

SQL Server Management Studio (SSMS): SQL Server Management Studio (SSMS) is the primary database management tool for SQL Server database servers. It is a graphical user interface (GUI) and a Transact-SQL scripting interface for managing the database engine component and databases. In addition, you can also use SSMS to manage different instances as well as cloud-based databases in Microsoft Azure SQL Database [15].

Microsoft Azure SQL Database: Microsoft Azure SQL Database is SQL Server, located in the cloud which provide the relational database with large-scaled capabilities on the platform with a high availability and keeps the load balance. The important thing is, there is no need to spend money on the equipment, licensing and pay for the costs that you use [16]. Using a subscription for academic purposes of Microsoft Azure, students will be able to use the cloud resource free of cost in the learning process and research work for one year.

ASP.NET MVC 5: ASP.NET MVC is an infrastructure for web application development built on the top of the advanced and popular .NET Framework. Infrastructure ASP.NET MVC relies heavily on the validated patterns and techniques of development that focuses on loosely-coupled architecture of the application and easily maintainable code. [17]. The built Bootstrap framework for creating an adaptive and extended interface can be a good addition to the version of MVC 5.

\section{Research Results Implementation}

\subsection{Setting up the local client-server system}

In order to understand the work of the cloud structure, firstly, it is necessary to get acquainted with the client-server system functions. In most cases, the internal process of cloud applications is based on a relational database, so some code is written in SQL or other queries [18], [19].

Thus, the practical part of the training course started with the creation of a clientserver system in the local network. Training on the traditional client-server system contributes to the formation of basic concepts of database activity and network 
processes. The stage of creating a local client-server system consisted of two parts. The first section was designed to configure server and client computers and implement network connections; the second section included the topics of creating database objects, developing queries and stored procedures.

The following steps were taken for the purpose of accomplishing this work, namely explanation the examples of using the local client-server system in the educational process in one classroom and formation of small groups of 3 or 4 students. Then each group set up a server and multiple client applications.

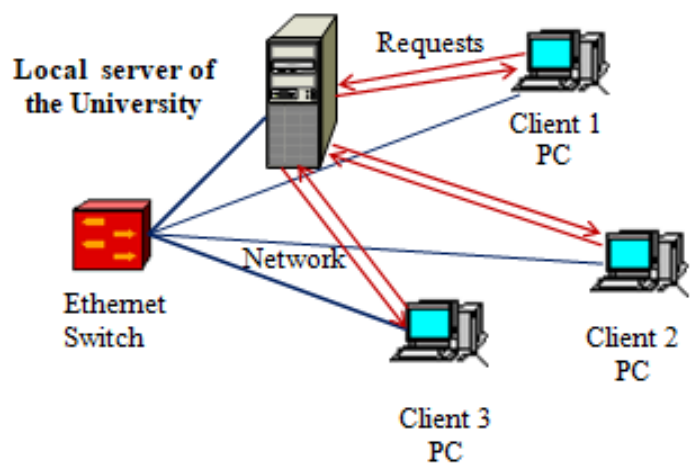

Fig. 2. Scheme of the local client-server system set up during the training process

In figure 2, a blue single line shows the work of a local network connected via Ethernet and a red double line indicates a server and client computers linked with a local network of University.

Program software and settings configured in the server computer are:

- $\quad$ Operating system Windows 10;

- $\quad$ MS SQL Server Express Edition 2016;

- $\quad$ SQL Server Management Studio.

1. Testing the integrity of the connection and the computers on the network (ping).

2. For the TCP/IP Protocol a remote connection was established by specifying port 1433 in the SQL Server Configuration Manager environment.

3. Inbound rules for protocols were created in the firewall. For the TCP/IP the rules were opened by port 1433, and 1434 for port UDP.

Program software and settings configured in client computers are:

- $\quad$ Operating system Windows 10;

- $\quad$ SQL Server Native Client 11.0;

- $\quad$ SQL Server Management Studio.

The required settings of the client computer are assigned.

The second part of the practical training was dedicated to the organization of work with databases. At this stage, the principles of the administrator and database users were defined, the main database of objects and data transmission to the server from client computers in the network were created. 
As a result, students were able to implement steps to configure client-server systems in the local network. As follows, the practical development of concepts and principles of the client-server system, network configuration and protocol assignment, communication with databases, query execution between the server and clients was carried out.

\subsection{Using Microsoft Azure cloud resources in academic activities for implementing a training course}

Cloud computing can provide innovative processes in business as well as in public and educational fields. The system, created on the basis of Microsoft Azure cloud resource. It is recognized as a leader in this field, can help students to gain knowledge that does not depend on the geography, increase hardware capacity and ensure the data security and privacy [20]. Advanced cloud computing coverage allows us to train students in new different ways and helps to manage the projects and large workloads. In this way, students and teachers can use applications without installing it on their computers and get access from any computer connected to the Internet [21].

Deployment of the remote server in the Microsoft Azure SQL Database cloud: In the second stage of practical work, students and teachers of L.N.Gumilyev Eurasian National University and Kh.Dosmukhamedov Atyrau State University were connected to the Microsoft Azure SQL Database. Figure 3 demonstrates the structure that was used for academic purposes in the preparation of Computer Science students. Students could connect to the remote server being in and out of the classroom. Azure for the students subscription was used for academic purposes. 


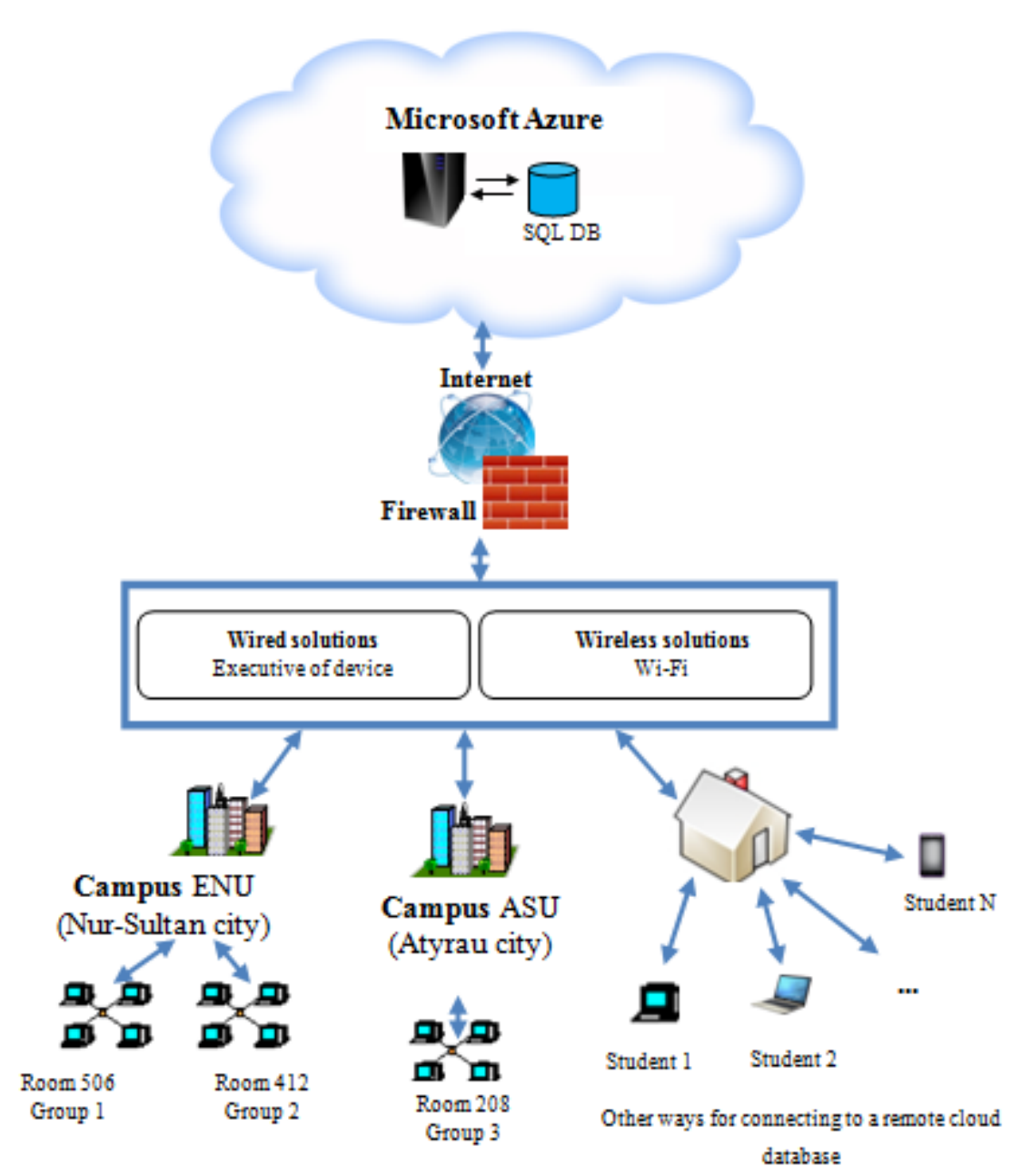

Fig. 3. Use of MS Azure resources in the development of a remote disk space and the structure of its academic implementation

Microsoft Azure SQL Database helps to run a flexible and mobile remote server that is scalable and widely available. Flexible to use interface helps to deploy the server and database without much effort and configuration. Figure 4 shows the window of creating the database and deploying servers in the cloud resource. The ways of deployment of Microsoft Azure SQL Database in the learning process in L. N.Gumilyev Eurasian National University are presented below. In this case, a server located in Western Europe was selected (Figure 4). 


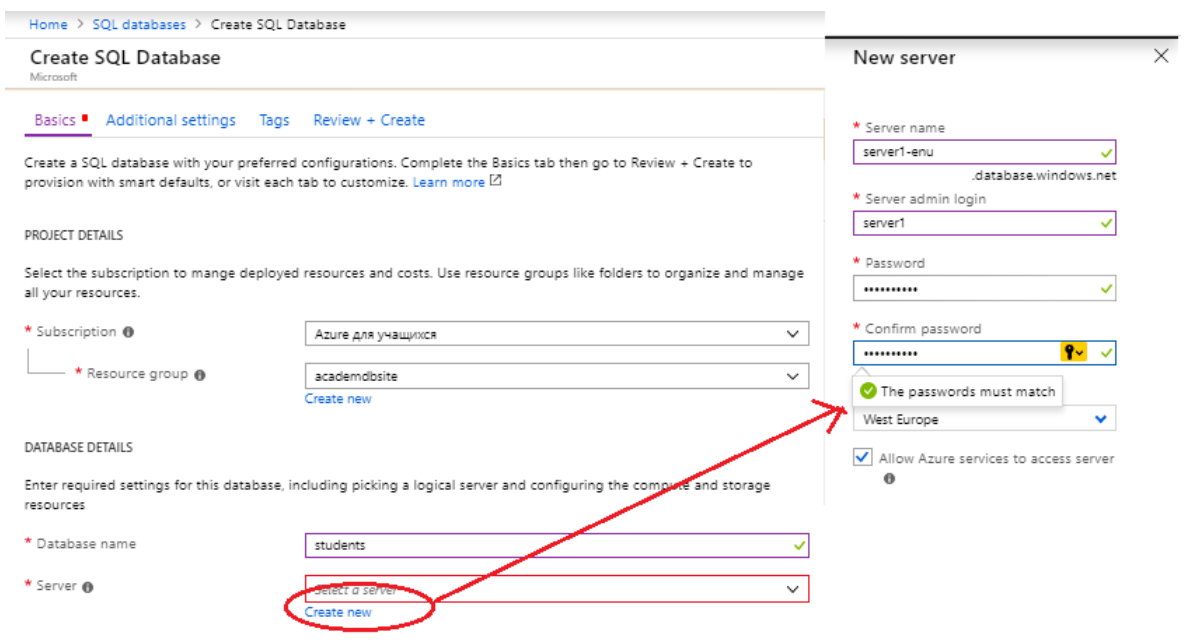

Fig. 4. Deployment of the remote server (server1-enu) in the cloud

Students created their own cloud SQL server and database. Besides, creating a new database in the cloud, there were established three replicas of that database to ensure high availability. This leads to the solution of the problem of data loss and ensures the privacy and security.

The tables, views and stored procedures created by us in the Azure portal are supported by the SSMS GUI. Figure 5 shows the window for connecting to server1enu in the cloud via SSMS. To open the server for use, it is needed to specify the names of a server and a login.

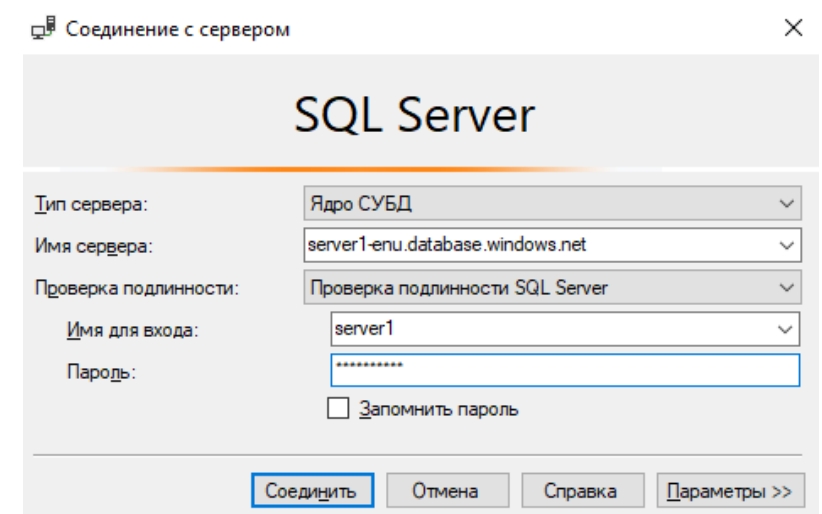

Fig. 5. Window for connecting to server1-enu in the cloud via SSMS

Database geo-replication: Performing replication provides the data reliability, availability and helps to improve the system performance. In a Microsoft Azure SQL 
Database cloud resource, the geo-replication command helps to create a copy of the database in any target region listed. The following figure 6 illustrates the geographic location of the target data regions for geo-replication.

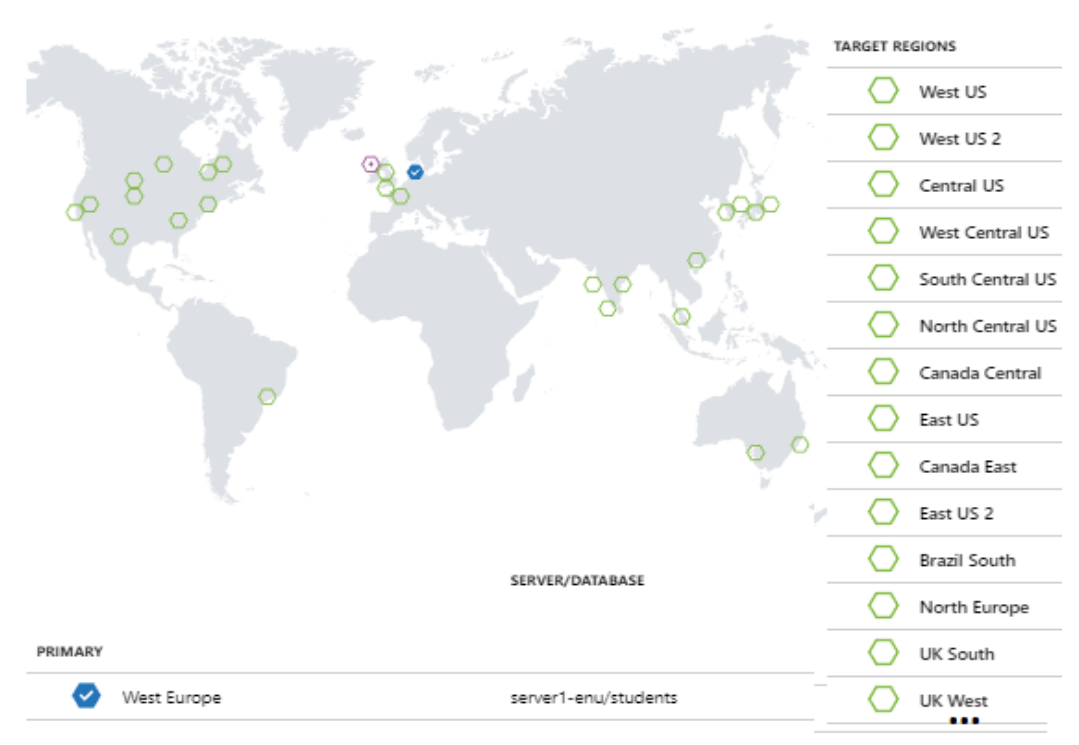

Fig. 6. General Scheme of the location of the target data processing regions including the chosen server

In the given example, the replication was organized for the server1-enu database created by us, located on a remote server in the Western Europe. A replicated copy of the database was selected as a server in the Eastern Europe.

Within this topic, the students were acquainted with the capabilities of cloud solutions, subscribed to a cloud resource, created servers and database in the cloud using Microsoft Azure SQL Database. They learned how to create the database objects and replicate of the data for their future program projects.

\subsection{Collaborative use of local and remote servers based on the principle of the hybrid cloud}

The third stage of the improved content of training course was addressed to link local and cloud servers based on the principle hybrid cloud. That principle allowed combining the capabilities of local and clouding servers. On the one hand, it was possible to attract flexibly external resources as required, and reduced the infrastructure costs. On the other hand, it retained full control over the data and applications that was not necessary to give out. Thus, we faced the challenge of integrating the data from different sources. To solve the integration problem, the query connections based on the hybrid cloud principle were created between local 
server MS SQL prepared at the first stage of the research task and the remote cloud server Microsoft Azure SQL Database (figure 7).

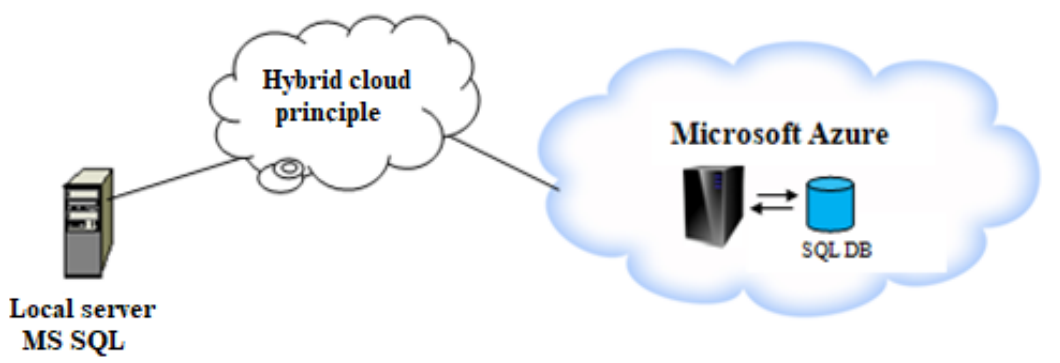

Fig. 7. Organized communication based on the principles of hybrid cloud

The following parameters and software are required to implement the hybrid cloud principle:

1. Local server MS SQL 2016;

2. Graphical user interface SSMS;

3. Account in Microsoft Azure:

4. Remote server in Microsoft Azure SQL Database.

SSMS was used to manage the distributed data received from two servers. After logging in an instance of SQL Express in LAPTOP, the command linked with the server was executed for the establish communication with Microsoft Azure cloud server at the address "server1-enu.database.windows.net".

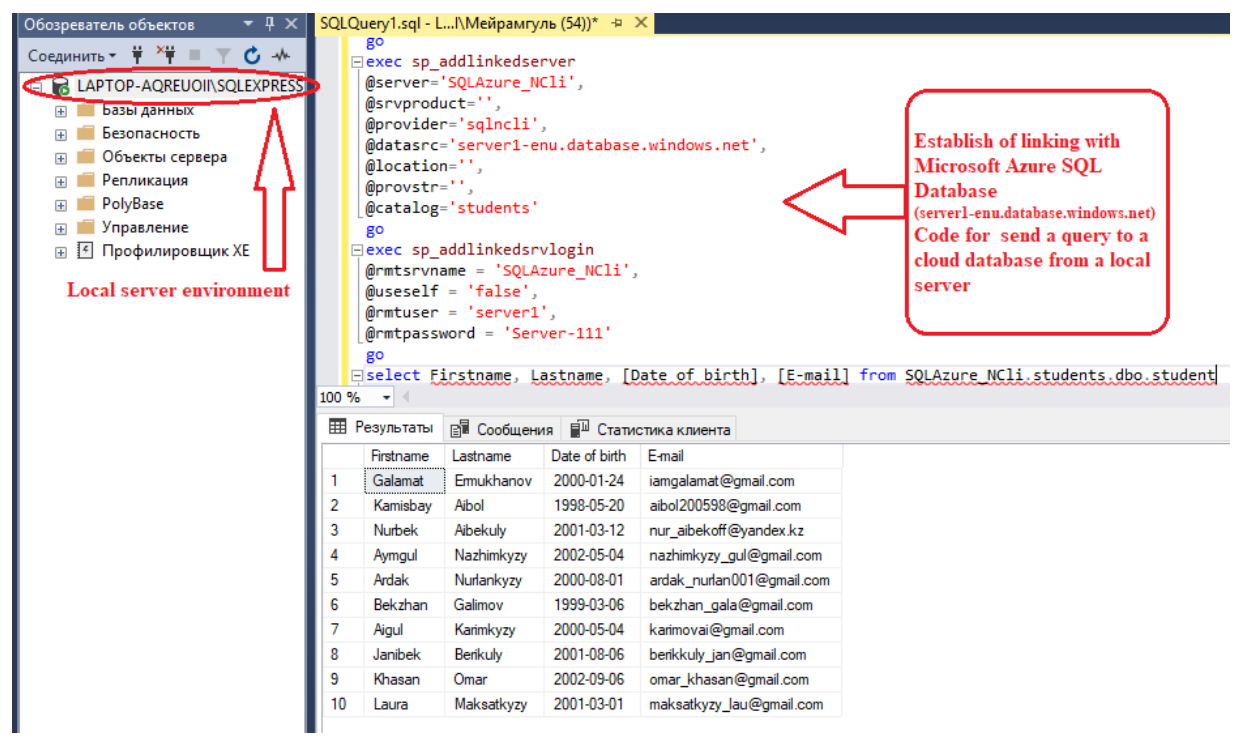

Fig. 8. Creation of the distributed queries in the linked servers utilizing of hybrid cloud 
Paper-Improving the Content of a Client-server Technology Training Course: Set up and Collaborative...

After the established communication between servers, the distributed heterogeneous queries and stored procedures were performed.

\subsection{Web applications using ASP.NET MVC 5 Framework development}

Joining the configured servers with the web client interface, ASP.NET MVC 5 Framework technology was used. Complex via IIS, MS SQL Server and ASP.NET had positive aspects as it had a single developer acting through Microsoft and accordingly was well integrated with each other [22].Web applications development through ASP.NETMVC 5 Framework was done using the C\# programming language in Visual Studio. In the MVC technology, the carcass of web interface consisted of the model-view-controller parts.

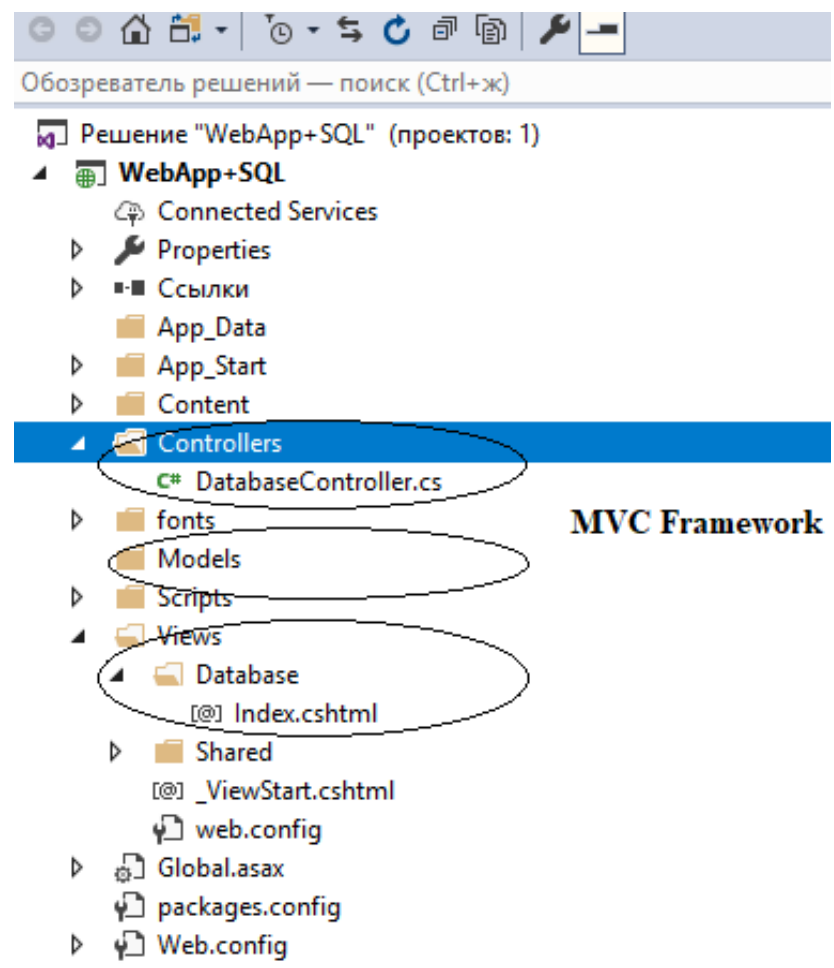

Fig. 9. Framework of MVC for web application 
Paper-Improving the Content of a Client-server Technology Training Course: Set up and Collaborative...

\subsection{The level of updates and improving the content of the training course}

It can be stated that the updated curriculum of university educational programs and its components for training future specialists for professional work is an important issue. The indicators presented in Table 1 were developed by us during the research.

Table 1. Percentage of updated topics in the course content

\begin{tabular}{|l|c|}
\multicolumn{1}{|c|}{ Topics for improving the content of the training course } & $\begin{array}{c}\text { \% of up- } \\
\text { dating }\end{array}$ \\
\hline $\begin{array}{l}\text { Familiarization with the modern hardware and software capabilities of client-server } \\
\text { technology. Cloud technologies as an extended version of client-server technology. }\end{array}$ & $20 \%$ \\
\hline Working with MS SQL Server 2016 platform components and management tools. & $30 \%$ \\
\hline Tools and capabilities of the SQL Server Management Studio graphical user interface. & $30 \%$ \\
\hline Organising of local network communication. Assignment of the protocols. & $10 \%$ \\
\hline Support of server and client settings. & $30 \%$ \\
\hline Execution of the client queries using T-SQL on the local network. & $15 \%$ \\
\hline $\begin{array}{l}\text { Creation of the web applications using the ASP.NET MVC Framework in local area } \\
\text { network }\end{array}$ & $40 \%$ \\
\hline $\begin{array}{l}\text { Use of cloud solutions in academic activities. Subscription in Microsoft Azure cloud } \\
\text { resource. }\end{array}$ & $20 \%$ \\
\hline $\begin{array}{l}\text { Deployment of server and creation of the database on Microsoft Azure SQL Database } \\
\text { cloud resource. }\end{array}$ & $80 \%$ \\
\hline $\begin{array}{l}\text { Establishment of communication between local and cloud servers based on the hybrid } \\
\text { cloud principles. }\end{array}$ & $80 \%$ \\
\hline \begin{tabular}{l} 
Implementation of the distributed, heterogeneous queries in the linked servers. \\
\hline
\end{tabular} & $80 \%$ \\
\hline
\end{tabular}

Overall, the different levels of percentages in Table 1 are explained as follows: the training course was a subject in the educational program of Computer Science major. The results also shows that improving the content of the training course, the work on filling in and updating existing topics was increased by $10-40 \%$ and a number of new topics introduced were $80 \%$. Students had the general information about the cloud technologies but those topics were introduced for the first time in the content of the course. The educational and methodical complex by new and updated topics were developed and implemented for the educational process.

\section{Conclusion}

It is important to note that organizing a client-server system in a local network, the form of installation and server configuration, implementation of network communication are the complex tasks. The transition of the production organizations to the use of the cloud technologies lies in avoiding the technical work but not in purchasing the licensed programs, spending money only for the used resources and implementing the information systems necessary for their activities. As an effective 
method of implementation, the hybrid cloud principle is able to store sensitive data on its local servers and host a large amount of database content on the cloud server, in which many users are present.

It is also worth mentioning that in our research we separately stopped on each of these directions, prepared methodical recommendations, educational and methodical complexes for the training course for its introduction in the educational process. Thus, the training course with the improved content would help students to improve their theoretical knowledge on the client-server technology as well as practical skills in the use of the modern systems. In our turn, we hope that this will contribute to the formation of a competitive specialist.

\section{References}

[1] Thomas, M. A., Redmond, R. T., Weistroffer, H. R. (2009). Moving To the Cloud: Transitioning From Client-Server to Service Architecture. Journal of Service Science (JSS), 2(1): 1-10.https://doi.org/10.19030/jss.v2i1.4286

[2] Nikulchev, E., Pluzhnik, E., Biryukov, D., Lukyanchikov, O. (2015). Designing applications in a hybrid cloud.Contemporary Engineering Sciences, 8(21): 963-970. http://dx.doi.org/10.12988/ces.2015.57214

[3] Serik, M., Mukhambetova, M. (2017). Klient-server technologiiasynyn oqytyly jagdaiyna sholy [A Review condition of teaching to client-server technology]. Abai Kazakh National Pedagogical University BULLETIN Ser. Physical \& Mathematical Sciences, 1 (61): 315318. [in Kazakh].

[4] Kravets, O. JA. (2013). Individualization of training in informatics of technical college students: theoretical bases. American Journal of Pedagogy and Education, 2(2): 19-25.

[5] Stillman R., Peslak, A. (2009). The Complexities of Effectively Teaching Client-Server System Development. Information systems Education Journal, 7(22): 1-10.

[6] Radchenko, G. I. (2012). Raspredelennyye vychislitelnyye sistemy [Distributed computing systems], Chelyabinsk: photo Artist, pp. 41-49.[in Russian].

[7] Wang, J. (2017). Cloud Computing Technologies in Writing Class: Factors Influencing Students' Learning Experience. Turkish Online Journal of Distance Education, 18 (3): 197-213.https://dx.doi.org/10.17718/tojde.328954

[8] Serik, M., Karelkhan, N., Sadvakassova, A.,Sarzhanova G.(2017). Improvement of students' training in parallel and cloud computing. Espacios, 38 (60): 3-13. http://www.revistaespacios.com/a17v38n60/17386003.html

[9] Liu, J., Fu, R. (2018). Development of an Accounting Skills Simulation Practice System Based on the B/S Architecture. International Journal of Emerging Technologies in Learning (iJET), 13(10): 134-145.https://doi.org/10.3991/ijet.v13i10.9459

[10] Chang, M., Zhang, M. (2019). Architecture Design of Datacenter for Cloud English Education Platform. International Journal of Emerging Technologies in Learning, 14(1). 24-33.https://doi.org/10.3991/ijet.v13i10.9459

[11] Sadvakassova A.K., Serik, M. (2017). Cloud technologies in educational system of Republic of Kazakhstan. Journal of Theoretical and Information Technology..95(11): 2434-2441. http://www.jatit.org/volumes/ninetyfive11.php

[12] Srinivasan, A., Quadir, M. A., \&Vijayakumar, V. (2015). Hybrid Cloud for Educational Sector. Procedia Computer Science, 50: 37-41. 
Paper-Improving the Content of a Client-server Technology Training Course: Set up and Collaborative...

[13] Pluzhnik, E., Nikulchev, E., Payain, S. (2014). Optimal control of applications for hybrid cloud services. In 2014 IEEE World Congress on Services. IEEE.pp. 458-461.

[14] Varga, S., Cherry, D., D'Antoni, J. (2016). Introducing Microsoft SQL Server 2016: Mission-Critical Applications, Deeper Insights, Hyperscale Cloud. Microsoft Press.

[15] Osetrova I.S. (2016)Administrirovaniye v MS SQL Server 2014 [Administration of MS SQL Server 2014]. SPb: University of ITMO, pp. 25-30. [in Russian]

[16] Krishnan S. (2010) Programming Windows Azure: Programming the Microsoft Cloud. O'Reilly Media, Inc.

[17] Chadwick, D., Panda, H., Snyder, T. (2013). ASP. NETMVC 4.Razrabotka realnykh vebprilozheniy s pomoshchyu ASP. NET MVC [ASP. NETMVC 4. Developing real-world web applications with ASP.NET MVC]. Litres. Hayes, B. (2008). Cloud computing. Communications of the ACM, 51(7), pp 9-11.

[18] Hayes, B. (2008). Cloud computing. Communications of the ACM, 51(7): 9-11.

[19] Serik, M., Karelkhan, N., Kultan, J., Zulpykhar, Zh. Setting up and implementation of the parallel computing cluster in education. International Journal of Emerging Technologies in Learning (iJET), 14(6): 4-17. https://doi.org/10.3991/ijet.v14i06.9736

[20] Morse N. V., Kuzminskaya E. G. (2011). Pedagogical aspects of cloud computing. Information technologies, 9: 20-29.[in Ukrainian]

[21] Saidhbi S. (2012). A cloud computing framework for Ethiopian Higher Education Institutions. IOSR Journal of Computer engineering, 6(6): 01-09.

[22] Grishchenko P. L. (2013). Prakticheskiye aspekty umensheniya nekotorykh riskov ITAutsorsinga [Practical aspects of reducing some risks of IT Outsourcing], 6: 365-370. [in Russian]

\section{Authors}

Meruert Serik professor, doctor of pedagogical sciences, Department of Computer Science, Faculty of Information Technology, L.N.Gumilyev Eurasian National University, Nur-Sultan, Kazakhstan. E-mail: serik_meruerts@mail.ru

Meiramgul Mukhambetova doctoral student, Department of Computer Science, Faculty of Information Technology, L.N.Gumilyev Eurasian National University, Nur-Sultan, Kazakhstan. E-mail: mukhambetovamj@gmail.com

Alibek Yeskermessuly PhD, Head of department of Computer Science, Faculty of Natural Science and Informatization, Arkalyk State Pedagogical Institute named after Y.Altynsarin, Arkalyk, Kazakhstan, E-mail: aleke1410@gmail.com

Article submitted 2019-04-10. Resubmitted 2019-08-12. Final acceptance 2019-08-12. Final version published as submitted by the authors. 